\title{
4D MAME Models for Live-Cell Imaging of Interactions Between Breast Tumor Cells and Their Microenvironment
}

\author{
B.F. Sloane, M. Sameni, A. Anbalagan, M.B. Olive, N. Shokoohi, K. Moin and R. Mattingly
}

Department of Pharmacology and Barbara Ann Karmanos Cancer Institute, Wayne State University School of Medicine, Detroit, MI 48201

We have a longstanding interest in how the interactions between tumors and their microenvironment impact the malignant progression of breast carcinomas. Our studies are focused on proteolytic pathways and their modulation by signaling pathways in the transition from preinvasive ductal carcinoma in situ (DCIS) to invasive ductal carcinoma (IDC). The functions of proteases in cancer are dynamic and much broader than simply degradation of extracellular matrices during tumor invasion and metastasis. Proteases from tumor-associated cells (e.g., fibroblasts, inflammatory cells, endothelial cells) as well as proteases from tumor cells contribute to 'tumor proteolysis'. Furthermore, the functions of any one protease are not constant in tumors, but may differ during the progression to neoplasia as well as from one stage of cancer to another. 'Tumor proteolysis' is often considered to result primarily from the activity of MMPs and serine proteases with the latter participating as a result of their ability to activate MMPs. Nonetheless, invasive processes in lower organisms such as parasites use cysteine cathepsins, suggesting that the human counterparts of these enzymes might also be involved in invasive processes during pathophysiologies [1].

We have concentrated on analyzing the roles in cancer of the cysteine cathepsin family of cysteine proteases and their endogenous inhibitors with a particular focus on cathepsin B. We and others have documented elevated expression at the transcript and protein level of cysteine cathepsins, including cathepsin B, in a wide variety of human tumors [2]. This elevated expression is found in tumor cells as well as in tumor-associated cells, e.g., macrophages and fibroblasts. Furthermore, we have previously shown that in tumors and tumor cells cathepsin B, normally a lysosomal protease, is also expressed at the cell surface [1]. Whether these increases in expression and altered localization are reflective of functional roles for cysteine cathepsins is for the most part an open question since there is limited knowledge of the critical substrates cleaved in vivo or in situ by any given protease. To assess functional roles for proteases requires the capability of assessing protease activity and inhibition of that activity rather than just the levels of expression of the proteases and activators or inhibitors of those proteases. Since proteases are often highly expressed at the invading edges of tumors, techniques that allow one to localize the sites of protease activity would be optimal. Furthermore, as no single protease or single protease family acts alone to enhance tumor progression, techniques that can delineate interactions among proteases and identify proteolytic pathways would increase our understanding.

To these ends, our laboratory has pioneered the development of techniques for functional imaging of protease activity associated with live cells [3] and of 3- and 4-D co-culture models that recapitulate the architecture of mammary tumors in the context of their microenvironment, i.e., MAME (mammary architecture and microenvironment engineering) models [4,5]. Use of these techniques and models in concert with various types of imaging probes has allowed us to image and quantify total protease activity, to image and quantify extracellular protease activity, to image and quantify intracellular protease activity, to discriminate activities of proteases or protease classes and to follow 
the dynamics of proteolysis associated with tumor cells alone and tumor cells interacting with other cells of the tumor microenvironment. In our studies to date, we have analyzed various combinations of breast epithelial cell variants, breast carcinoma cells, breast [myo]fibroblasts, breast myoepithelial cells, monocytes/ macrophages and endothelial cells of blood vessel and lymphatic origin. We grow these co-cultures in $3 \mathrm{D}$ matrices of reconstituted basement membrane $(\mathrm{rBM}) \pm$ stromal collagen I and image by confocal microscopy over time (4D) changes in cellular morphology, migration and invasion and interactions among the various cell types. In parallel, we follow the degradation of quenched-fluorescent protein substrates [3]. Our results indicate that MAME models can be used to study crosstalk among proteolytic and signaling pathways associated with tumor:microenvironment interactions that modulate the transition from DCIS to invasive ductal carcinoma. We hypothesize that these analyses will identify potential targets for therapeutic intervention and candidate biomarkers to distinguish preinvasive lesions that will rapidly progress to invasive lesions from lesions that remain indolent.

\section{References}

[1] M.M. Mohamed and B.F. Sloane, Nature Rev. Cancer 6 (2006) 764.

[2] C. Jedeszko and B.F. Sloane, Biol. Chem. 385 (2004) 1017.

[3] C. Jedeszko et al., Curr. Protoc. Cell Biol. 39 (2008) 4.20.1.

[4] M. Sameni et al., Clin. Exp. Metastasis 26 (2009) 299.

[5] D. Cavallo-Medved et al., Exp. Cell Res. 315 (2009) 1234.

[6] This research was supported by U.S. Public Health Service Grants CA 56586 and 131990 and a Breast Cancer Center of Excellence from the Department of Defense (DAMD17-02-1-0693). The Microscopy, Imaging and Cytometry Resources Core is supported by National Institutes of Health Center Grant P30CA22453.

A

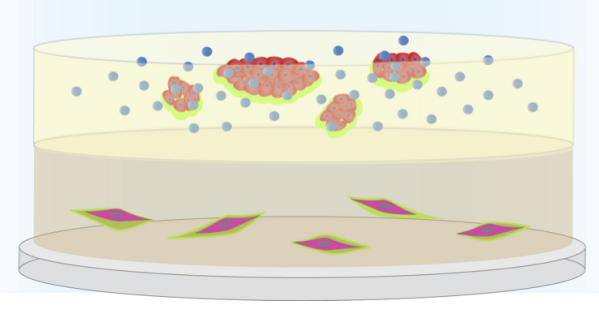

B

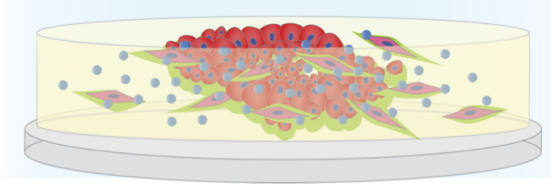

FIG. 1. Schematic of MAME co-culture models used for live-cell imaging. A) Tripartite MAME model: Coverslips are coated with collagen I containing DQ-collagen $\mathrm{I}^{\mathrm{TM}}$ (Invitrogen) and fibroblasts. A $2^{\text {nd }}$ layer of $\mathrm{rBM}$ containing DQ-collagen $\mathrm{IV}^{\mathrm{TM}}$ (Invitrogen) is added and DCIS cells plated on top along with leukocytes. The cultures are then overlaid with a $3^{\text {rd }}$ layer of $2 \% \mathrm{rBM}$, which also is included in subsequent changes of media. B) Mixed MAME: Coverslips are coated with rBM containing DQ-collagen $\mathrm{IV}^{\mathrm{TM}}$ and fibroblasts and DCIS cells plated on top along with leukocytes. Co-cultures are imaged by confocal microscopy and resulting optical sections reconstructed in 3D using Volocity (Perkin Elmer) software. 